\title{
A Rare Case of Classical Hodgkin Lymphoma Diagnosed 10 Years after Liver Transplant
}

\author{
$\begin{array}{llll}\text { L. Zhang } & \text { a,b } & \text { R. Pereira Mestre } & \text { F. Bihl } \\ \text { A.d } & \text { M. Bühler } & \text { B. Vannata }\end{array}$ \\ A. Stathis ${ }^{a}$
}

${ }^{a}$ Oncology Institute of Southern Switzerland, Bellinzona, Switzerland; ${ }^{\text {b Institute of }}$ Hematology, Union Hospital, Tongji Medical College, Huazhong University of Science and Technology, Wuhan, China; ${ }^{C}$ Cantonal Hepatology Service, Cantonal Hospital Ticino, Bellinzona, Switzerland; ${ }^{\mathrm{d}}$ Department of Gastroenterology and Hepatology, University Hospital Geneva, Geneva, Switzerland; ${ }^{e}$ Cantonal Institute of Pathology,

Locarno, Switzerland

\section{Keywords}

Hodgkin lymphoma · Posttransplant lymphoproliferative disorders · Transplantation ·

Hepatitis C virus · Epstein-Barr virus · Immunosuppression

\begin{abstract}
Posttransplant lymphoproliferative disorders (PTLD) represent a rare and potentially lifethreatening complication after liver transplantation. Classical Hodgkin lymphoma ( $\mathrm{cHL})$, with an incidence of approximately $1.8-3.4 \%$ of all PTLD cases, represents a minority of PTLD, mainly presenting as a late transplant complication. The main risk factors for the development of PTLD are Epstein-Barr virus (EBV) infection and intensive immunosuppression. However, other risk factors like hepatitis $C$ virus may, together with EBV infection, contribute to the development of PTLD. Here we present a case of late-onset EBV-positive $\mathrm{CHL}$ that occurred 10 years after an unrelated donor liver transplantation. To our knowledge, this is the first report of $\mathrm{CHL}$ occurring with such a long interval after liver transplantation. Given the low incidence of CHL PTLD, there is little information regarding pathology, clinical characteristics, and management of this disease. The development of individual, risk-adapted treatments may improve the long-term outcome of CHL PTLD.




\section{Case Reports in Oncology}

\section{Introduction}

Posttransplant lymphoproliferative disorders (PTLD) are lymphoid or plasmocytic proliferations that develop as a consequence of immunosuppression following solid organ or hematopoietic stem cell transplant, with an incidence ranging from 1 to 10\% [1]. Most cases are associated with Epstein-Barr virus (EBV)-mediated proliferation of B cells and transformation in lymphoblastic cells in a setting of decreased T-cell immune surveillance. The incidence of PTLD following liver transplantation is approximately $2-3 \%$, being the lowest in comparison with the incidence after kidney, lung, cardiac or intestinal transplants $[1,2]$. Among the various histological categories of PTLD, Classical Hodgkin lymphoma (cHL) is a late complication of transplantation and represents only 1.8-3.4\% of all PTLD cases, mostly arising after renal transplantation, with a median time to onset of 88 months after transplantation and very few cases described in the literature.

Here we present a case of late-onset EBV-positive cHL that occurred 10 years after an unrelated donor liver transplantation. To our knowledge, this is the first report of cHL occurring with such a long interval after liver transplantation.

\section{Case Report}

A 61-year-old male presented with multiple superficial lymphadenopathies and weight loss of $15 \mathrm{~kg}$. His medical history was significant for previous hepatitis B, chronic obstructive pulmonary disease (COPD) with pulmonary emphysema related to tobacco use, and heavy alcohol consumption. The patient also had a chronic hepatitis $\mathrm{C}$ virus (HCV) infection leading to cirrhosis and hepatocellular carcinoma treated with unrelated donor liver transplantation, which was performed 10 years prior to the onset of the present symptoms. Immunosuppressive treatment following liver transplant consisted initially of mycophenlote mophetyl (MMF) and tacrolimus (FK-506) without particular infectious complications. MMF was suspended around 5 years after transplantation and monotherapy with low-dose FK506 (plasma titers 3-6 $\mu \mathrm{mg} / \mathrm{L}$ ) was maintained. For HCV reinfection of the graft with evolution towards advanced fibrosis, the patient was then treated with sofosbuvir/daclatasvir plus ribavirin for 6 months with sustained virological response in 2015.

Laboratory examinations showed increased $\beta_{2}$-microglobulin $(3.8 \mathrm{mg} / \mathrm{L})$ and erythrocyte sedimentation rate $(70 \mathrm{~mm} / \mathrm{h})$. The patient was negative for hepatitis $B$ surface antigen, hepatitis C RNA, and human immunodeficiency virus, but positive for hepatitis B core antibody. EBV DNA by PCR was positive with a low viremia $(131 \mathrm{IU} / \mathrm{mL})$ at the first presentation but negative in a repeated test before treatment start.

A total-body CT scan confirmed multiple lymphadenopathy. A biopsy of an axillary lymph node showed subverted architecture and the presence of Hodgkin and ReedSternberg (RS) cells in a background consisting of mixed inflammatory cells. Immunohistochemistry showed expression of CD30, CD15, EBV-LMP1, and PAX5 (weak) in Hodgkin and RS cells, without expression of CD45, CD20, and CD5. EBER (EBV-encoded RNA) hybridization was positive. Staging procedures were completed with 18F-FDG PET/CT showing intensive pathological FDG uptake in multiple abdominal lymph nodes, both below and above the diaphragm, with bone marrow and spleen involvement. ECG and echocardiography were normal, and the pulmonary function test showed a mild decrease in the diffusion parameters. 


\section{Case Reports in Oncology}

The final diagnosis was cHL PTLD stage IVB. Tacrolimus was suspended and the patient started chemotherapy with 2 cycles of ABVD regimen (doxorubicin, bleomycin, vinblastine, and dacarbazine) via intramuscular injection of half-dose bleomycin. To reduce the risk of bleomycin-induced lung toxicity (BLT) in consideration of the COPD, lung emphysema, and active smoking, the patient was treated with AVD (omitting bleomycin) in the next 4 cycles. An interim PET/CT after cycle 2 showed complete metabolic response in all sites of previous involvement (Deauville score 3). During chemotherapy, the patient presented severe symptomatic hyponatremia with corresponding hypoosmolality $(114 \mathrm{mmol} / \mathrm{L})$ in the context of documented adrenal insufficiency (confirmed by ACTH stimulation test) and was treated with hydrocortisone replacement. Another complication during treatment was a painful bone fracture of vertebrae L1-L3 and T7-T11 in the context of severe osteoporosis secondary to prolonged steroid therapy and treated with percutaneous vertebroplasty (bone biopsy excluded cHL).

The end of treatment PET/CT showed sustained nodal and spleen complete metabolic response but with a lung and esophageal FDG uptake of uncertain significance. In consideration of these findings an esophago-gastro-duodenoscopy was performed with documentation of an esophageal diverticulum and gastritis not related to Helicobacter pylori. A bronchoscopy with bronchoalveolar lavage was negative for viral, fungal, or bacterial infections and did not reveal any neoplastic cells on cytology. The pulmonary findings were in regression in a follow-up imaging with PET/CT and no longer suspect for secondary malignancy. At 7 months after the end of chemotherapy the patient is in complete remission.

\section{Discussion}

The incidence of PTLD is correlated with the type of organ transplantation, the intensity of immunosuppression, the occurrence of viral infections, and the age at transplantation. Although PTLD are histologically heterogeneous, abundant evidence suggests that EBV plays a critical role in their pathophysiology. EBV can induce blastic transformation and uncontrolled B-cell proliferation. It has been reported that negative EBV serology before transplant is significantly associated with increased risk of non-Hodgkin lymphoma in kidney transplant recipients [3]. Nevertheless, a high EBV load in transplant recipients does not predict the development of PTLD [4].

Despite the known causative role of EBV in PTLD development, the effectiveness of antiviral agents in the treatment or prophylaxis of EBV-related PTLD is limited. They have been reported to inhibit lytic EBV DNA replication in vitro, but have no effect against B cells in latent state or on the proliferation of EBV-transformed B cells. On the other hand, antiviral therapy following transplantation may have a role as prophylaxis, and it has been reported to reduce the risk of PTLD in renal recipients by up to 83\% [5]. However, this finding was not confirmed in a multicenter retrospective study of 44,828 kidney transplant recipients [6].

HCV infection represents a risk factor for non-Hodgkin lymphoma among an immunocompetent population, particularly for B-cell lymphomas. HCV infection increases the risk of developing PTLD, irrespective of the type of immunosuppression used [7]. Chronic HCV infection may lead to the development of PTLD by lymphoid stimulation and clonal expansion of B cells [8]. However, Stamataki et al. [9] found that HCV could infect B cells, but B cells were not sufficient to support HCV replication. Coinfection with HCV and another virus might be needed for HCV infection and replication in B cells. The ability of EBV to support 


\section{Case Reports in Oncology}

HCV replication has been reported [10], with some evidence suggesting a possible synergistic role of HCV/EBV coinfection in promoting B-cell blastic transformation [11]. In addition, it has been observed that HCV envelope protein E2 delivers a costimulatory signal to B cells in association with EBV [11].

The prognosis of PTLD is variable, partly due to the heterogeneity of the disease, which ranges from benign hyperplasia to aggressive malignant lymphoma, with estimated survival rates ranging from 25 to $60 \%$ [12]. A recent study from the Mayo Clinic proposed a multivariate prognostic model in which a performance status score of 3-4, monomorphic disease, and grafted organ involvement predicted poor prognosis [13]. Other negative risk factors proposed include EBV-negative status of the recipient, late onset of disease, disease involving multiple sites, advanced age, stage, elevated LDH, severe organ dysfunction, and central nervous system involvement. Treatment decisions are made based on the pathological subtype, grade, and site of the tumor as well as on adequate assessment of the patient's clinical state, including transplanted organ function. Relatively little information on the prognosis of cHL PTLD patients is available. However, several cases published have shown that cHL PTLD patients can tolerate standard chemotherapy/radiotherapy, achieve complete remission, and have a long-term disease-free survival [12]. The prognosis of cHL seems more favorable than other monomorphic PTLD.

Because RS-like cells may be seen in early, polymorphic, and some monomorphic PTLD, the diagnosis of cHL must be based on both classical morphological, and immunophenotypic features. PTLD showing RS morphology but with strong expression of CD20 and negative for CD15 should be diagnosed as HL-like PTLD and classified as monomorphic B-cell PTLD. Distinction from cHL PTLD may be important for clinical management and prognosis.

Although there is no standard treatment for CHL PTLD, the first most common management is a reduction of immunosuppressive agents. As for the presented case, suspension of tacrolimus was carried out according to normal hepatic function and then followed with chemotherapy. Fortunately he was treated for HCV infection shortly before with a virological response. The patient was therefore treated with the ABVD chemotherapy regimen, with a reduced bleomycin dose for the first 2 cycles and without bleomycin for cycles 3-6. BLT has been described in HL patients. The incidence of BLT in older HL patients ranges from 5 to $36 \%$, with mortality up to $27 \%$ [14]. However, the GHSG HD13 trial showed that omission of bleomycin results in an increased risk of relapses compared with standard ABVD chemotherapy [15]. On the other hand, there is a high risk of BLT in older HL patients receiving more than 2 cycles of ABVD [14]. In addition to older age, other risk factors, including renal deficiency, radiation, underlying lung disease, smoking history, and granulocyte colonystimulating factor support, also increase the risk of BLT. Therefore, we decided to give 2 cycles of ABVD with half-dose bleomycin via intramuscular injection because of lung emphysema. Given the good response on the interim PET-CT, the patient will continue chemotherapy with AVD regimens.

\section{Conclusion}

cHL occurring after liver transplantation is a rare late transplant complication. The prognosis of cHL is more favorable compared with that of monomorphic PTLD. There is no general consensus on the best therapeutic approaches for cHL PTLD patients, although the most common approaches are immunosuppression reduction, chemotherapy, and radiotherapy, and maybe antiviral therapy for concomitant HCV infection. Treatment of cHL PTLD 
is compatible with consideration of the agent's toxicity, the patient's graft function, and his/her capacity to tolerate therapy. The development of individual risk-adapted treatments may improve long-term outcomes for cHL patients.

\section{Statement of Ethics}

The authors have no ethical conflicts to disclose.

\section{Disclosure Statement}

The authors have no conflicts of interest to declare.

\section{References}

1 Opelz G, Dohler B: Lymphomas after solid organ transplantation: a collaborative transplant study report. Am J Transplant 2004;4:222-230.

2 Taylor AL, Marcus R, Bradley JA: Post-transplant lymphoproliferative disorders (PTLD) after solid organ transplantation. Crit Rev Oncol Hematol 2005;56:155-167.

-3 Opelz G, Daniel V, Naujokat C, Dohler B: Epidemiology of pretransplant EBV and CMV serostatus in relation to posttransplant non-Hodgkin lymphoma. Transplantation 2009;88:962-967.

-4 Green M, Webber SA: EBV viral load monitoring: unanswered questions. Am J Transplant 2002;2:894895.

5 Funch DP, Walker AM, Schneider G, Ziyadeh NJ, Pescovitz MD: Ganciclovir and acyclovir reduce the risk of post-transplant lymphoproliferative disorder in renal transplant recipients. Am J Transplant 2005;5:2894-2900.

-6 Opelz G, Daniel V, Naujokat C, Fickenscher H, Dohler B: Effect of cytomegalovirus prophylaxis with immunoglobulin or with antiviral drugs on post-transplant non-Hodgkin lymphoma: a multicentre retrospective analysis. Lancet Oncol 2007;8:212-218.

-7 McLaughlin K, Wajstaub S, Marotta P, et al: Increased risk for posttransplant lymphoproliferative disease in recipients of liver transplants with hepatitis C. Liver Transpl 2000;6:570-574.

8 Morton LM, Landgren 0, Chatterjee N, et al: Hepatitis C virus infection and risk of posttransplantation lymphoproliferative disorder among solid organ transplant recipients. Blood 2007;110:4599-4605.

-9 Stamataki Z, Shannon-Lowe C, Shaw J, et al: Hepatitis C virus association with peripheral blood B lymphocytes potentiates viral infection of liver-derived hepatoma cells. Blood 2009;113:585-593.

10 Petrova M, Kamburov V, Nikolovska D, Kosseva O, Nikolova M, Krastev Z: Epstein-Barr virus: is there any contribution to chronic hepatitis B and C? Liver Int 2010;30:488-489.

11 Park GB, Kim D, Park SJ, et al: Pre-stimulation of CD81 expression by resting B cells increases proliferation following EBV infection, but the overexpression of CD81 induces the apoptosis of EBVtransformed B cells. Int J Mol Med 2015;36:1464-1478.

12 Leblond V, Dhedin N, Mamzer Bruneel MF, et al: Identification of prognostic factors in 61 patients with posttransplantation lymphoproliferative disorders. J Clin Oncol 2001;19:772-778.

13 Ghobrial IM, Habermann TM, Maurer MJ, et al: Prognostic analysis for survival in adult solid organ transplant recipients with post-transplantation lymphoproliferative disorders. J Clin Oncol 2005;23:7574-7582.

14 Boll B, Goergen H, Behringer K, et al: Bleomycin in older early-stage favorable Hodgkin lymphoma patients: analysis of the German Hodgkin Study Group (GHSG) HD10 and HD13 trials. Blood 2016;127:2189-2192.

15 Behringer K, Goergen H, Hitz F, et al: Omission of dacarbazine or bleomycin, or both, from the ABVD regimen in treatment of early-stage favourable Hodgkin's lymphoma (GHSG HD13): an open-label, randomised, non-inferiority trial. Lancet 2015;385:1418-1427. 